\title{
Dispersion Management and Optical Phase Conjugation in Optical Transmission Links with a Randomly Distributed Single-Mode Fiber Length
}

\author{
Seong-Real Lee*, Member, KIICE
}

Department of Marine Information and Communication Engineering, Mokpo National Maritime University, Mokpo 530-729, Korea

\begin{abstract}
Suppressing or mitigating signal distortion due to group velocity dispersion and optical Kerr effects is necessary in ultrahigh speed and long-haul wavelength division multiplexing (WDM) transmission systems. Dispersion management (DM), optical phase conjugation (OPC), and the combination of these two are promising techniques to compensate for signal distortion. In this paper, to implement a flexible optical WDM network, a new optical link configuration with a randomly distributed single-mode fiber (SMF) length and fixed residual dispersion per span in the combination of DM and OPC is proposed and investigated. The simulation results show that the best net residual dispersion (NRD) in the proposed optical links is $+10 \mathrm{ps} / \mathrm{nm}$, which is independent of pre- and postcompensation. The effective launch power of the WDM channel is increased more in the optical links with $\mathrm{NRD}=+10 \mathrm{ps} / \mathrm{nm}$ controlled by only precompensation. Furthermore, the system performance difference between the proposed optical link configuration with the best NRD and the conventional optical link with uniform distribution of the SMF length had little significance. Consequently, it is confirmed that the proposed optical link configuration with the best NRD is effective and useful for implementing a reconfigurable long-haul WDM network.
\end{abstract}

Index Terms: Dispersion management, Optical phase conjugation, Residual dispersion per span, Net residual dispersion, Pre/Postcompensation.

\section{INTRODUCTION}

Group-velocity dispersion (GVD) and optical nonlinearity are the major limiting factors in high-speed long-distance fiber-optic transmissions, such as wavelength division multiplexing (WDM) systems with a $40 \mathrm{Gbps}$ channel data rate [1]. In such systems, as the transmission distance and span length under the required optical signal-to-noise ratio are increased, the launch power has to be increased. Thus, the nonlinear Kerr effect becomes one of the most important impairments. Optical phase conjugation is a promising technique to com- pensate for these nonlinear impairments [2-4]. The concept is based on optical phase conjugation in an optical phase conjugator (OPC) placed at the middle of a fiber transmission link. The phase conjugated signal generated by the OPC carries the same data as the original signal but the signal spectrum is inverted. This technique offers unique advantages over other competitive methods such as data rate and modulation format transparency, ultra-fast responses, simultaneous multi-channel compensation, and compensation of nonlinear distortions. It is independent of the transmission fiber's dispersion property as long as the same type of fiber is used

Received 21 October 2012, Revised 18 November 2012, Accepted 30 November 2012

*Corresponding Author Seong-Real Lee (E-mail: reallee@mmu.ac.kr, Tel: +82-62-240-7264)

Department of Marine Information and Communication Engineering, Mokpo National Maritime University, 91 Haeyangdaehang-ro, Mokpo 530-729, Korea. 
for both halves of the transmission link.

To minimize the impact of distortion due to GVD only, a dispersion map involving optical pre- and postcompensation as a dispersion management (DM) technique has also been developed [5-7]. Pre- and postcompensation are defined as dispersion compensation using dispersion compensating fiber (DCF) after the transmitter and before the receiver, respectively. In the case of a dispersion map applied to every fiber span, residual dispersion per span (RDPS) is defined as the dispersion accumulated in each fiber span, and net residual dispersion (NRD) is defined as the total dispersion accumulated at the end of the transmission link. These quantities are key parameters for designing a transmission system with high performance. Generally, NRD is decided by controlling preor postcompensation and RDPS. The most advanced DCFs are even capable of slope-matching compensation, namely, compensating the dispersion and the dispersion slope of the transmission fiber simultaneously. However, the DM technique using DCF is used only in optical links without nonlinear effects on optical signals. That is, DM can compensate for distorted optical signals due to only GVD.

In the optical phase conjugation technique, compensation for nonlinearity by OPC is limited by the asymmetry of the strength of the Kerr effect along the fiber with respect to the OPC position. However, there are a number of techniques for overcoming this drawback. For example, optimizing the OPC position [8, 9] or combining appropriate dispersion mapping [8], [10, 11] has been proposed recently. In order to suppress the nonlinearity impairments to a large extent, the system parameters of an OPC link, such as the location of the OPC or the dispersion map, need be optimized.

In a previous work, the author has also shown that a 960 Gbps (40 Gbps $\times 24$ channels) WDM transmission system with good receive performance was implemented by applying the combined DM and OPC into optical links [12]. In this research, the basic scheme of DM is that the system NRD is controlled by precompensation using DCF of the first span, postcompensation using DCF of the last span, and RDPS of the same value in the rest of the fiber spans. The single-mode fiber (SMF) length of every fiber spans between an erbium-doped fiber amplifier (EDFA) are assumed to be equal, for simplicity of optical link configuration in the previous work. However, the SMF length needs to be unlimited for flexible implementation of the optical network topology. As far as the author knows, the analysis and assessment of a WDM transmission link to which OPC and DM are applied, with random distribution of the SMF length in every fiber span, have not been reported yet.

Therefore, in this work, optimal NRDs are induced by assessing the eye opening penalty (EOP) of the worst channel among the 24 WDM channels in optical links with randomly distributed SMF length and a fixed $150 \mathrm{ps} / \mathrm{nm}$ of RDPS in every fiber span. Also, the effective launching powers of WDM channels in the optical links with the induced optimal NRD are presented to meet the design requirements of the flexible ultra-high optical transmission network.

\section{WDM TRANSMISSION MODEL}

\section{A. Modeling of Optical Links}

The optical transmission link configuration investigated in this research is shown in Fig. 1. The total transmission links consist of 14 fiber spans, which include SMF and DCF. The SMF length of all of the spans are designed to be fixed at $l_{S M F}=80 \mathrm{~km}$ for uniform distribution, and on the other hand, to be variable for random distribution. All of the SMFs of the above two cases were characterized by attenuation coefficient $\alpha_{S M F}=0.2 \mathrm{~dB} / \mathrm{km}$, dispersion coefficient $D_{S M F}=17 \mathrm{ps} / \mathrm{nm} / \mathrm{km}$, and nonlinear coefficient $\gamma_{S M F}=1.41$ $\mathrm{W}^{-1} \mathrm{~km}^{-1}$ at $1,550 \mathrm{~nm}$.

In order to generate symmetric distribution of dispersion with respect to OPC, the DCFs are placed before each SMF in earlier half section from the transmitter (Tx) to the OPC, while the DCFs are placed after each SMF in the latter half section from the OPC to the receiver ( $\mathrm{Rx})$, as illustrated in Fig. 1. The DCF length $l_{D C F}$ was fixed at $12.1 \mathrm{~km}$, which makes the RDPS $150 \mathrm{ps} / \mathrm{nm}$ in the uniform distribution of SMF length. On the other hand, $l_{D C F}$ was also varied by the selected SMF length for each fiber span, but the RDPS of all of the fiber spans was maintained at $150 \mathrm{ps} / \mathrm{nm}$ in the optical links with randomly distributed SMF lengths. All of the DCFs of the above two cases were characterized by dispersion coefficient $D_{D C F}=-100 \mathrm{ps} / \mathrm{nm} / \mathrm{km}$, attenuation coefficient $\alpha_{D C F}=0.4 \mathrm{~dB} / \mathrm{km}$, and nonlinear coefficient $\gamma_{D C F}=$ $4.83 \mathrm{~W}^{-1} \mathrm{~km}^{-1}$ at $1,550 \mathrm{~nm}$.

In this research, the averaged SMF lengths were assumed to be equal to $80 \mathrm{~km}$ in both transmission sections in the

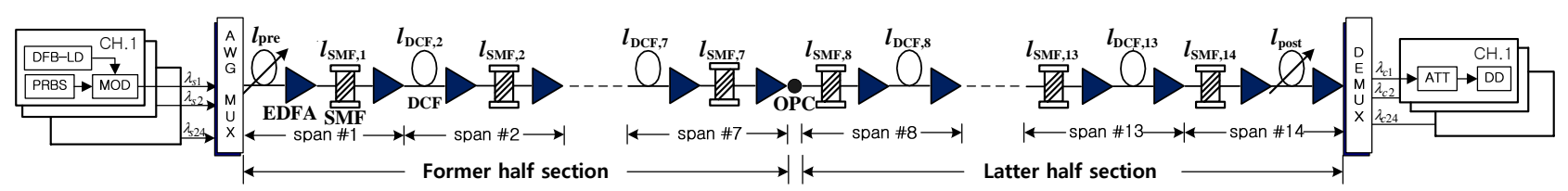

Fig. 1. A 960 Gbps WDM transmission system and optical link with optical phase conjugator (OPC) and dispersion management (DM) technique. EDFA: erbium-doped fiber amplifier, SMF: single-mode fiber, DCF: dispersion compensating fiber. 
case of random distribution. Namely, the SMF length of each fiber span was randomly selected to be one value among $50,60,70,80,90,100$, or $110 \mathrm{~km}$ in each half section; thus the averaged SMF length of one fiber span became $80 \mathrm{~km}$, according to $\Sigma l_{S M F, n} / 7$. As previously mentioned, in case of random distribution, $l_{D C F}$ depended on the selected SMF length for each fiber span according to $\left(l_{S M F} \cdot D_{S M F}-\right.$ RDPS $) /\left|D_{D C F}\right|$. For example, if the SMF length of an arbitrary fiber span had been selected to be 60 $\mathrm{km}$, then the DCF length was assigned to be $8.7 \mathrm{~km}$.

The NRD is controlled by pre- or postcompensation in both cases of uniform distribution and random distribution of SMF length, as plotted in Fig. 1. In case of determining the NRD by only precompensation, the NRD depends on the variable length of the first DCF, i.e., $l_{\text {pre }}$, the accumulated dispersion in the first SMF, and the total RDPS $(=150 \times 6$ $\mathrm{ps} / \mathrm{nm}$ ) in the earlier half section, when the total accumulated dispersion in the latter half section has been fixed to be 0 $\mathrm{ps} / \mathrm{nm}$. On the other hand, when the NRD is determined by only postcompensation, it depends on the variable length of the last DCF, i.e., $l_{\text {post }}$, the accumulated dispersion in the last SMF, and the total RDPS in the latter half section when the total accumulated dispersion in the former half section has been fixed to be $0 \mathrm{ps} / \mathrm{nm}$.

\section{B. Modeling of WDM Transmission System}

The WDM system consisted of $\mathrm{Tx}, \mathrm{Rx}$, the dispersion managed optical links, and the OPC nearby the midway of total transmission length. The Tx (Fig. 1) was assumed to be a distributed feedback laser diode (DFB-LD). The center wavelength of the DFB-LD was assumed to be 1550-1568.4 $\mathrm{nm}$ by spacing of $100 \mathrm{GHz}(0.8 \mathrm{~nm})$ based on ITU-T recommendation G.694.1. Thus, if each wavelength was allocated for one WDM channel, the total wavelength considered corresponds to 24-channel WDM transmission. The DFB-LD was externally modulated by an independent 40 Gbps $128\left(=2^{7}\right)$ pseudo random bit sequence (PRBS). The modulation format from the external optical modulator was assumed to be return-to-zero (RZ), and the output electric field of the RZ format was assumed to be a secondorder super-Gaussian pulse with a $10 \mathrm{~dB}$ extinction ratio, duty cycle of 0.5 , and chirp-free.

The nonlinear medium of OPC midway along the total transmission length is highly nonlinear dispersion-shifted fiber (HNL-DSF). The parameters of OPC using HNL-DSF are as follows: loss of HNL-DSF $\alpha_{0}=0.61 \mathrm{~dB} / \mathrm{km}$, nonlinear coefficient of HNL-DSF $\gamma_{0}=20.4 \mathrm{~W}^{-1} \mathrm{~km}^{-1}$, length of HNLDSF $z_{0}=0.75 \mathrm{~km}$, zero dispersion wavelength of HNL-DSF $\lambda_{0}=1550 \mathrm{~nm}$, dispersion slope $d D_{0} / d \lambda=0.032 \mathrm{ps} / \mathrm{nm}^{2} / \mathrm{km}$, pump light power $P_{p}=18.5 \mathrm{dBm}$, and pump light wavelength $\lambda_{\mathrm{p}}=1549.75 \mathrm{~nm}$. The $3-\mathrm{dB}$ bandwidth of conversion efficiency $\eta$ of the OPC was set at $48 \mathrm{~nm}(1526-1574 \mathrm{~nm})$,

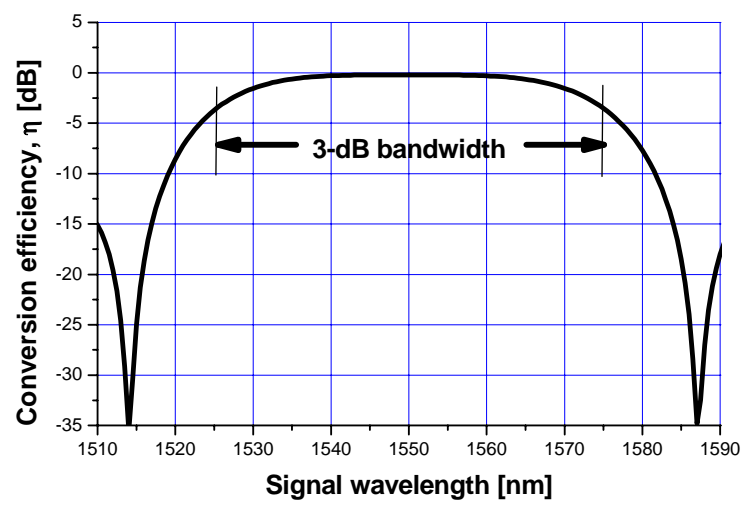

Fig. 2. Conversion efficiency of optical phase conjugator.

as shown in Fig. 2. The signal wavelengths were converted to $1549.5-1528.5 \mathrm{~nm}$ (these are called the conjugated wavelength) through OPC. Thus, the 24 signal wavelengths and these conjugated wavelengths belonged within the $3-\mathrm{dB}$ bandwidth of $\eta$.

The conjugated wavelengths are sent into the $\mathrm{Rx}$ of direct detection. The Rx consists of the pre-amplifier of EDFA with $5 \mathrm{~dB}$ noise figure, the optical filter of $1 \mathrm{~nm}$ bandwidth, PIN diode, pulse shaping filter (Butterworth filter), and the decision circuit. The receiver bandwidth is assumed to be at $0.65 \times$ bit-rate [2].

\section{NUMERICAL ASSESSMENT METHOD}

The propagation of the signal in a lossy, dispersive, and nonlinear medium can be expressed by the nonlinear Schrödinger equation, assuming a slowly varying envelope approximation [1]:

$$
\begin{aligned}
& \frac{\partial A_{j}}{\partial z}=-\frac{\alpha}{2} A_{j}-\frac{i}{2} \beta_{2 j} \frac{\partial^{2} A_{j}}{\partial T^{2}}+\frac{1}{6} \beta_{3 j} \frac{\partial^{3} A_{j}}{\partial T^{3}} \\
& +i \gamma_{j}\left|A_{j}\right|^{2} A_{j}+2 i \gamma_{j}\left|A_{k}\right|^{2} A_{j},
\end{aligned}
$$

where $j, k=1,2, \ldots, 24(j \neq k), A_{j}$ represents the complex amplitude of the signal of the $j$-th channel, $z$ is the propagation distance, $\beta_{2 j}$ is the GVD, $\beta_{3 j}$ is third-order dispersion, $\gamma_{j}$ is nonlinear coefficient, and $T=t-z / v_{j}$ is the time measured in a retarded frame. The last two terms of (1) induce selfphase modulation and cross-phase modulation (XPM), respectively. The effects of XPM on WDM signals decrease as the fiber dispersion increases [9]. The XPM effect is neglected in this work because the dispersion coefficient of fiber in this research is assumed to be $17 \mathrm{ps} / \mathrm{nm} / \mathrm{km}$. The numerical approach of (1) is completed by using the splitstep Fourier method [1].

The EOP was used to assess the system performance of the receiving WDM signals in this work, as shown in the 
following equation:

$$
E O P[d B]=10 \log _{10} \frac{E O_{r e c}}{E O_{b t b}},
$$

where $E O_{\text {rec }}$ and $E O_{b t b}$ are the eye opening (EO) of the receiving optical pulse and $\mathrm{EO}$ of the input optical pulse, respectively. EO is defined as the following equation:

$$
2 P_{a v} /\left(P_{1, \min }-P_{0, \max }\right)
$$

where $P_{a v}$ is the averaged power of the optical signals, and $P_{1, \min }$ and $P_{0, \max }$ are the minimum power of the ' 1 ' optical pulse and the maximum power of the ' 0 ' optical pulse, respectively.

\section{SIMULATION AND DISCUSSION}

There are many random distribution cases of SMF length. It is difficult to assess the system performance of the overall cases because it is a time consuming process. Therefore, in this work, 10 cases of random distribution are considered for an accurate and simple assessment.

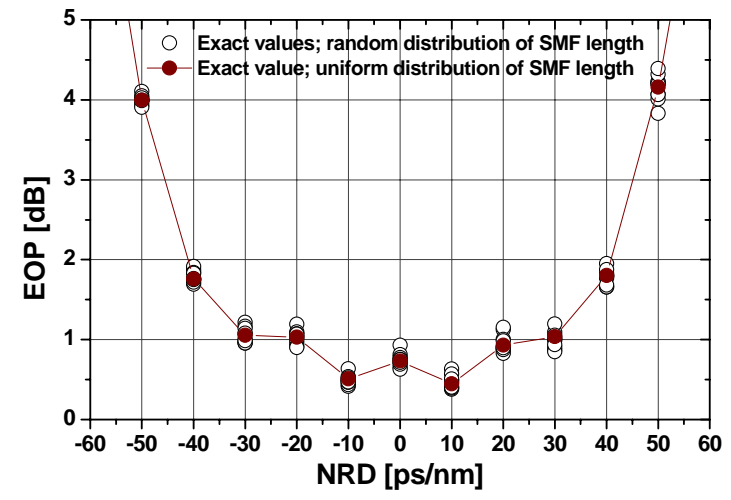

(a)

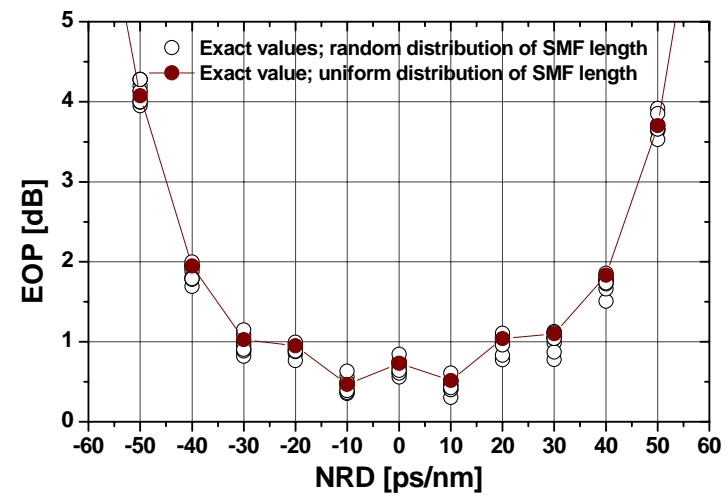

(b)

Fig. 3. Eye opening penalty (EOP) of the worst channel versus the net residual dispersion (NRD) controlled by precompensation (a) and postcompensation (b). SMF: single-mode fiber.
Fig. 3a and $\mathrm{b}$ illustrate EOPs of the worst channel among the $24 \mathrm{WDM}$ channels with launching power of $-2 \mathrm{dBm}$ as a function of the NRD controlled by pre- and postcompensation, respectively, in optical transmission links with the randomly distributed SMF length. Fig. 3 also includes the EOP obtained from optical links with a uniform distribution of the SMF length in order to compare the two. It is shown that the EOPs depend on the NRD as well as the SMF length distribution. It is confirmed that the optimal NRDs were found to be $10 \mathrm{ps} / \mathrm{nm}$ or $-10 \mathrm{ps} / \mathrm{nm}$ in the optical link controlled by pre- and postcompensation for uniform and random distributions of the SMF length because the EOPs were the smallest values at these NRDs. Through analysis of the results of Fig. 3, it is also confirmed that the EOP depends upon the pattern of the SMF length random distribution. Namely, in the optical links with the randomly distributed SMF length, it is predicted that the deviation of the EOP will depend on the launch power of the WDM channels as well as the distribution pattern of the SMF length.

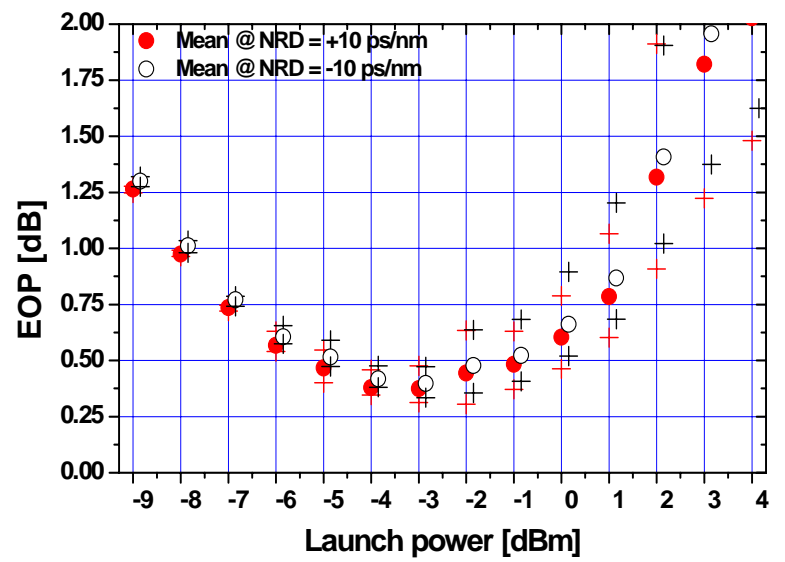

(a)

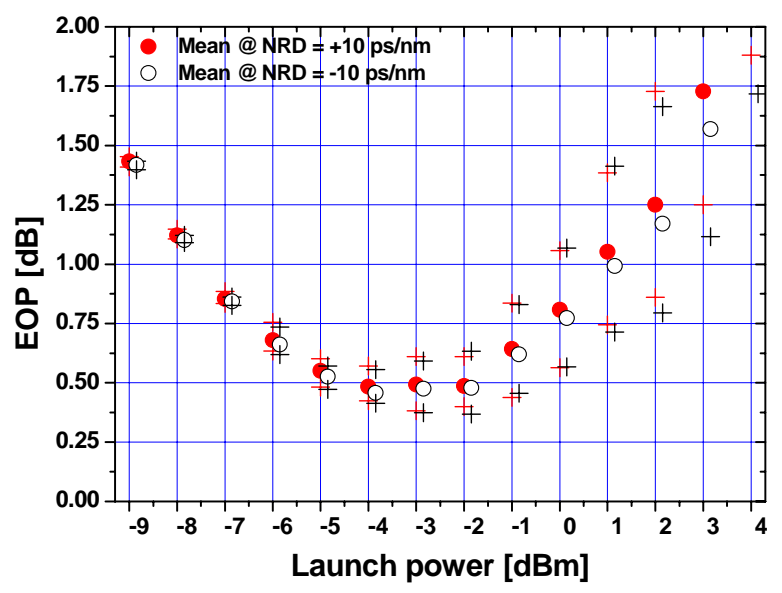

(b)

Fig. 4. Mean eye opening penalty (EOP) and EOP deviation of worst channel versus launching power at optimal net residual dispersion (NRD) controlled by precompensation (a) and postcompensation (b). 
Fig. 4a and b show the worst channel's mean EOPs and EOP deviations as a function of the channel launch power at the optimal NRD obtained. In Fig. 4, the symbols ' $\bullet$ ' and ' $\circ$ ' express the mean EOP obtained from 10 random patterns at optimal NRD, and ' + ' is the deviation of these EOPs. From Fig. 4 , it is confirmed that the deviation of the EOP as well as the mean EOPs increase as the channel launch powers increase in the range above $-4 \mathrm{dBm}$. Namely, EOPs are less affected by random distribution of the SMF length at a relatively small launch power.

It is also shown that, in optical links with optimal NRD controlled by precompensation, i.e., in Fig. $4 \mathrm{a}$, the mean EOPs and EOP deviations obtained at the NRD of $10 \mathrm{ps} / \mathrm{nm}$ are less than those obtained at the NRD of $-10 \mathrm{ps} / \mathrm{nm}$, for the entire launch power range considered. In addition, in optical links with optimal NRD controlled by postcompensation, i.e., in Fig. 4b, though the mean EOPs and EOP deviations obtained at the NRD of $-10 \mathrm{ps} / \mathrm{nm}$ are slightly less than those obtained at the NRD of $10 \mathrm{ps} / \mathrm{nm}$, the differences between the two cases are negligible, in the range below -2 $\mathrm{dBm}$. On the other hand, in Fig. 4b, the mean EOPs and EOP deviations obtained at the NRD of $10 \mathrm{ps} / \mathrm{nm}$ are less than those obtained at the NRD of $-10 \mathrm{ps} / \mathrm{nm}$, for the range above $-3 \mathrm{dBm}$. Therefore, it is confirmed that the best NRD is $+10 \mathrm{ps} / \mathrm{nm}$ in the optical link with the randomly distributed SMF length, which is independent of pre- and postcompensation required for deciding the NRD.

Fig. 5a and $\mathrm{b}$ show the worst channel's EOPs as a function of the channel launch power at the obtained optimal NRD of $10 \mathrm{ps} / \mathrm{nm}$ and $-10 \mathrm{ps} / \mathrm{nm}$, which is controlled by pre- and postcompensation, respectively. If the criterion value of the EOP for excellent reception performance is selected to be $1 \mathrm{~dB}$ EOP, then the launch power resulting below $1 \mathrm{~dB}$ EOP is defined as the allowable launch power. In the optical link with $\mathrm{NRD}=10 \mathrm{ps} / \mathrm{nm}$ controlled by precompensation, the allowable launch power values are obtained to be -8 to $2.3 \mathrm{dBm}$ and -8 to $0.8 \mathrm{dBm}$ for the distribution pattern of SMF length resulting in the best system performance and the worst performance, respectively. Namely, at the high launch power range, the deviation in the effective launch power is almost $1.5 \mathrm{~dB}$ depending on the random distribution pattern of the SMF length.

On the other hand, in the optical link with NRD $=-10$ $\mathrm{ps} / \mathrm{nm}$ controlled by postcompensation, the allowable launch power values are obtained to be -7.7 to $2.6 \mathrm{dBm}$ and -7.6 to $0.3 \mathrm{dBm}$ for the distribution pattern of the SMF length resulting in the best system performance and the worst performance, respectively; namely, the deviation in the effective launch power is almost $2.9 \mathrm{~dB}$ in the high power range. Therefore, the deviation in the effective launch power depending on a random pattern in the optical link with -10 $\mathrm{ps} / \mathrm{nm}$ controlled by postcompensation is larger than that in the optical links with $10 \mathrm{ps} / \mathrm{nm}$ controlled by precompensation.

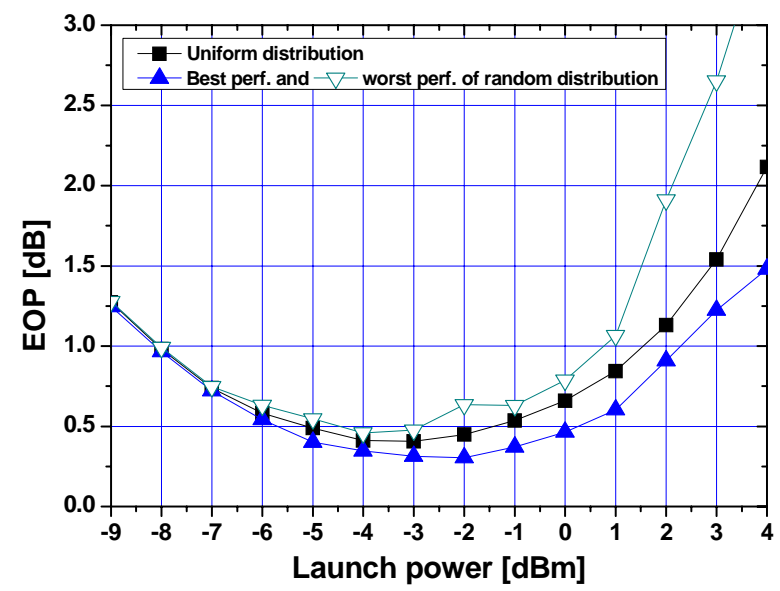

(a)

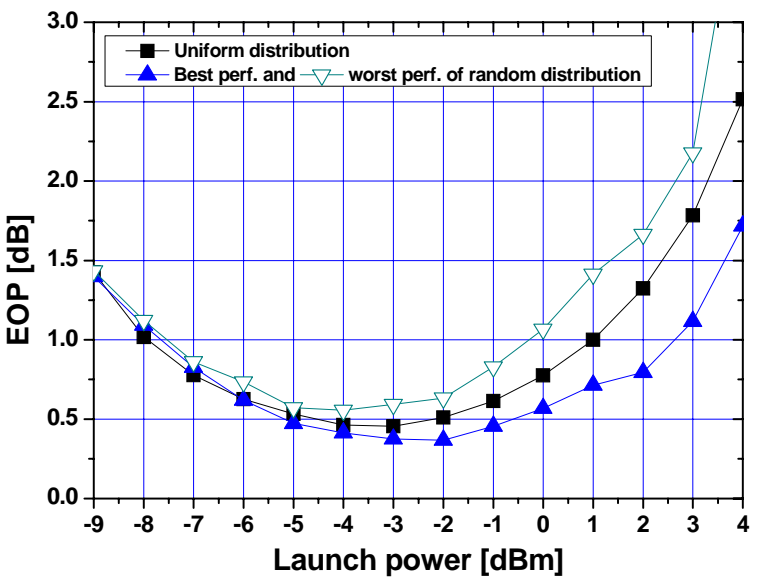

(b)

Fig. 5. Eye opening penalty (EOP) of the worst channel versus launching power at net residual dispersion (NRD) $=+10 \mathrm{ps} / \mathrm{nm}$ (a) and NRD = $-10 \mathrm{ps} / \mathrm{nm}(\mathrm{b})$

It is practically difficult to determine the specific pattern of random distribution of the SMF length for obtaining the best system performance. Thus, it is necessary to consider the worst case, that is, a random distribution pattern obtaining the worst system performance for implementing the flexible optical transmission links. From this viewpoint, the best NRD will be $10 \mathrm{ps} / \mathrm{nm}$ controlled by precompensation, and simultaneously, the launch power of the WDM channel will be from $-8 \mathrm{dBm}$ to almost $1 \mathrm{dBm}$ in optical links with a multi-span of the randomly distributed SMF length and the fixed RDPS.

\section{CONCLUSIONS}

In this paper, an optical link with a multi-span consisted of the randomly distributed SMF length and a fixed RDPS was proposed for implementing the flexible WDM transmis- 
sion network configuration. Also, in the proposed optical links, the parameters relating DM schemes and the parameters required for WDM transmission of high performance were investigated. The optimal NRD controlled by pre- or postcompensation, and the effective launch powers of WDM channels have been induced.

The simulation results show that the best NRD for optical links with the randomly distributed SMF length was 10 $\mathrm{ps} / \mathrm{nm}$, which is independent of pre- and postcompensation. From the perspective of the effective launch power, the best condition of the optical links for transmitting the widest power range is also NRD $=10 \mathrm{ps} / \mathrm{nm}$ controlled by precompensation. Furthermore, in the optimized optical links with the previously induced values, because the difference in the effective launch power from that in the optical links with uniform distribution was less than $1 \mathrm{~dB}$, namely, there is little significant difference between two optical links, it is confirmed that the proposed optical link, which consisted of the randomly distributed SMF length and fixed RDPS, which had the NRD of $10 \mathrm{ps} / \mathrm{nm}$, is an effective and useful optical link configuration.

\section{REFERENCES}

[1] G. P. Agrawal, Nonlinear Fiber Optics, 3rd ed. San Francisco, CA: Academic Press, 2001.

[2] G. P. Agrawal, Fiber-Optic Communication Systems, 3rd ed. New York, NY: Wiley, 2002.

[3] S. L. Jansen, D. van den Borne, B. Spinnler, S. Calabro, H. Suche, P. M. Krummrich, W. Sohler, G. D. Khoe, and H. de Waardt, "Optical phase conjugation for ultra long-haul phase-shift-keyed transmission," Journal of Lightwave Technology, vol. 24, no. 1, pp. 5464, 2006.
[4] X. Tang and Z. Wu, "Reduction of intrachannel nonlinearity using optical phase conjugation," IEEE Photonics Technology Letters, vol. 17, no. 9, pp. 1863-1865, 20015.

[5] X. Xiao, S. Gao, Y. Tian, and C. Yang, "Analytical optimization of the net residual dispersion in SPM-limited dispersion-managed systems," Journal of Lightwave Technology, vol. 24, no. 5, pp. 2038-2044, 2006.

[6] L. Gruner-Nielsen, M. Wandel, P. Kristensen, C. Jorgensen, L. V. Jorgensen, B. Edvold, B. Palsdottir, and D. Jakobsen, "Dispersioncompensating fibers," Journal off Lightwave Technology, vol. 23, no. 11, pp. 3566-3579, 2005.

[7] M. Suzuki and N. Edagawa, "Dispersion-managed high-capacity ultra-long-haul transmission," Journal of Lightwave Technology, vol. 21, no. 4, pp. 916-929, 2003.

[8] X. Xiao, C. Yang, S. Gao, and Y. Tian, "Partial compensation of Kerr nonlinearities by optical phase conjugation in optical fiber transmission systems without power symmetry," Optical Communication, vol. 265, no. 1, pp. 326-330, 2006.

[9] P. Minzioni, F. Alberti, and A. Schiffini, "Optimized link design for nonlinearity cancellation by optical phase conjugation," IEEE Photonics Technology Letters, vol. 16, no. 3, pp. 813-815, 2004.

[10] A. Chowdhury and R. J. Essiambre, "Optical phase conjugation and pseudolinear transmission," Optical Letters, vol. 29, no. 10, pp. 1105-1107, 2004.

[11] P. Minzioni and A. Schiffini, "Unifying theory of compensation techniques for intrachannel nonlinear effects," Optical Express, vol. 13, no. 21 , pp. 8460-8468, 2005.

[12] S. R. Lee, "Dispersion managed optical transmission links with optimized optical phase conjugator", International Journal of Maritime Information and Communication Science, vol. 7, no. 3, pp. 372-376, 2009.

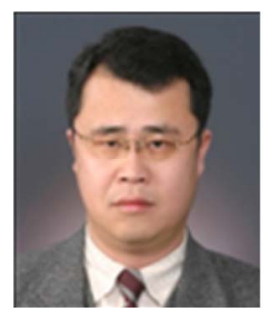

\section{Seong-Real Lee}

received the B.S., M.S., and Ph.D. degrees in telecommunication and information engineering from Hankuk Aviation University, Korea in 1990, 1992, and 2002, respectively. He is currently an associate professor of the Department of Marine Information and Communication Engineering at Mokpo National Maritime University. His research interests include optical WDM systems, optical soliton systems, and optical nonlinear effects. 